\title{
RECEPTOR INDUCING PROTEIN-2 (RIP-2) DEFICIENCY IS NEUROPROTECTIVE AGAINST HYPOXIC-ISCHEMIC BRAIN INJURY IN NEWBORN MICE
}

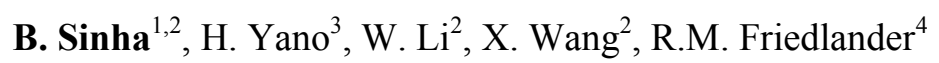 \\ ${ }^{1}$ Boston University School of Medicine, ${ }^{2}$ Neuroapoptosis Laboratory, Harvard Medical School, \\ ${ }^{3}$ Neurosurgery, University of Pittsburgh School of Medicine, Boston, MA, ${ }^{4}$ Neurosurgery, University of \\ Pittsburgh School of Medicine, Pittsburgh, PA, USA
}

Background and aims: The recent years have seen a shift in the management strategies of hypoxic ischemic (H-I) brain injury from being largely supportive to targeting the processes of ongoing injury. The apoptotic cell death pathway executed by caspases, have emerged as critical components of the pathophysiology of hypoxia-ischemia. In this study, we hypothesized that in neonatal mice with deletion of receptor inducing protein-2 (Rip-2), one of the important mediators of caspasel activation, the brain is protected against H-I injury relative to wild-type (WT) mice.

Methods: C57BL-6 Wild type+/+, Rip2 +/- and Rip2 -/- mice underwent unilateral carotid ligation under isofluorane anesthesia at postnatal day 8 (P8), followed by one hour of exposure to $8 \%$ oxygen. Pups were then returned to the dam until sacrifice at 1 week post H-I injury for histological staining and at earlier time points for western blot assay.

Results: Rip2 -/- mice $(n=13)$ had significant decrease $(p<0.005)$ in brain tissue loss at level of hippocampus compared to wild type mice $(n=12) 7$ days post H-I injury. Rip 2 was increased in WT mice following H-I injury. There was less caspase 3 expression in Rip2 KO mice compared to WT mice following H-I injury.

Conclusions: Our results suggest that Rip-2 deficiency protects the newborn mice from H-I injury. Understanding the mechanisms modulating cell death pathways in newborn H-I injury would help in development of therapies that can be appropriately targeted and offer hope to thousands of newborns with this devastating condition. 